\title{
Learning curve for endobronchial ultrasound-guided transbronchial needle aspiration: Experience of a community-based teaching hospital
}

\author{
HE WANG ${ }^{1,2}$, CYRIL D'CRUZ ${ }^{1}$, DAVID C. YAM ${ }^{1}$, LORIE M. DILLA ${ }^{1}$ and PATRICIA TSANG ${ }^{1}$ \\ ${ }^{1}$ Department of Laboratory Medicine and Pathology, Newark Beth Israel Medical Center, \\ Barnabas Health System, Newark, New Jersey 07112; ${ }^{2}$ Department of Pathology and Laboratory Medicine, \\ Temple University School of Medicine, Philadelphia, PA 19140, USA
}

Received September 4, 2013; Accepted May 14, 2014

DOI: $10.3892 / \mathrm{mmr} .2014 .2533$

\begin{abstract}
Endobronchial ultrasound-guided transbronchial needle aspiration (EBUS-TBNA) is a minimally invasive procedure. This procedure is useful for nodal staging of lung cancer and evaluating mediastinal lymphoma and granuloma. The present study was a retrospective analysis of our experience when EBUS-TBNA was initially implemented. A total of 112 lymph nodes/masses (51 patients) were divided into two groups: The first and second 8 months. In the first group, 33 lymph nodes/masses (16 patients) were biopsied and tumor diagnoses were made in $9 \%$ of the cases (three lymph nodes/masses). The material was adequate to produce a cell block for microscopic analysis in $42 \%$ of cases. Subsequent tissue diagnoses were available in 50\% of cases. Only one of the three malignant EBUS-TBNA diagnoses (33\%) was confirmed by histological examination. In the second 8 months, 79 lymph nodes (35 patients) were sampled. Tumor/granuloma diagnoses were achieved in $27 \%$ of the cases ( 21 nodes) $(\mathrm{P}=0.045$ versus the first 8 months) and the obtained material was adequate for producing a cell block in $90 \%$ of cases $(\mathrm{P}<0.001$ versus the first 8 months). Corresponding tissue diagnoses were available in 28\% of cases. Correlation of EBUS-TBNA and histological examination for tumor/granuloma diagnosis was $100 \%$ (12/12, $\mathrm{P}=0.029$ versus the first 8 months). Immunostains in the cell blocks indicated that all the metastatic adenocarcinomas were thyroid transcription factor- 1 (TTF-1) ${ }^{+}$and $\mathrm{p} 63^{-}$, and that all squamous cell carcinomas were TTF-1- $1^{-}$p63 $3^{+}$and cytokeratin 5/6 $(\mathrm{CK} 5 / 6)^{+}$. Eight granulomata were identified, of which five were positive for Acid-Fast Bacilli (AFB) stain and confirmed by culture or tissue biopsy. The remaining three granulomata were
\end{abstract}

Correspondence to: Dr He Wang, Department of Pathology and Laboratory Medicine, Temple University School of Medicine, 3401 N. Broad Street, Philadelphia, PA 19140, USA

E-mail: he.wang@tuhs.temple.edu

Key words: EBUS-TBNA, learning curve, mediastinal lymph nodes, TTF-1, p63
AFB-negative. EGFR/KRAS mutation analysis was conducted in cell blocks of five adenocarcinomas, of which all provided sufficient diagnostic material. The findings showed a steep learning curve when EBUS-TBNA was first adopted, reflected by an increased rate of tumor/granuloma diagnoses as well as an improved sample yield for cell block preparation in the second 8 months. TTF-1, p63 and CK5/6 were useful biomarkers for distinguishing metastatic lung carcinomas.

\section{Introduction}

The assessment of mediastinal adenopathy by non-invasive imaging modalities, such as computed tomography (CT) and positron emission tomography (PET), is a clinically useful technique. However the accuracy of these imaging modalities is usually insufficient to distinguish between benign and malignant lymph nodes for the purpose of determining subsequent treatment options. The sensitivity of CT and PET is between 60 and $85 \%$; while the specificity is between 79 and $90 \%(1,2)$. In current practice, pathological examination is necessary for PET-positive lymph nodes for mediastinal staging of lung cancer. Endobronchial ultrasound-guided transbronchial needle aspiration (EBUS-TBNA) is a minimally invasive procedure that was approved by the US Food and Drug Administration in March 2006. The effectiveness of EBUS-TBNA in mediastinal staging of lung carcinoma and in diagnosing central and peripheral lung masses, mediastinal involvement of lymphoma and isolated mediastinal lymphadenopathy has been previously reported (1-3). In addition to impressive sensitivity and specificity, EBUS-TBNA may be performed in the ambulatory care setting under sedation, the procedure is cost-effective, accessible to more lymph node stations, with less post-procedural complications $(2,3)$. Previous studies emphasized the importance of close communication between bronchoscopists, radiologists and pathologists for the success of EBUS-TBNA procedure $(4,5)$. However, the majority of these published data are from large tertiary medical centers with advanced stages of disease and greatly enlarged lymph nodes $(6,7)$. Conclusions from these studies may not represent the typical patient population in general practice. 
The present retrospective study reviewed our initial experience with EBUS-TBNA since its first introduction into the Newark Beth Israel Medical Center (Newark, NJ, USA), covering a period of 16 months. This study additionally discussed the efficient use of immunostaining with the limited material in cell blocks

\section{Materials and methods}

Patients. All patients with mediastinal or hilar lymphadenopathy or a mediastinal mass referred for EBUS-TBNA in the Newark Beth Israel Medical Center between January 2011 and April 2012 were enrolled in this study. This study was approved by the institutional Ethics Committee, and followed international and national regulations. All patients provided informed consent. The primary indications for TBNA were the sampling and diagnosis of enlarged lymph nodes of unknown origin and lung cancer staging. A chest radiograph was routinely performed in these patients, and selected patients also underwent a CT scan of the chest (plain and/or contrast enhanced).

EBUS-TBNA. Conventional flexible bronchoscopy (BF-7160 bronchoscope; Olympus,Ltd., Tokyo,Japan) was first performed by standard methodology to examine the tracheobronchial tree, followed by EBUS-TBNA using the new ultrasound biopsy bronchoscope (XBF-UC260F-OL8; Olympus, Ltd.). Both bronchoscopy procedures were performed by the same operator. Endobronchial ultrasound was performed using a prototype linear array ultrasonic bronchoscope. The instrument, similar to a standard bronchoscopic videoscope, had an outer diameter of $6.9 \mathrm{~mm}$, a $2.0-\mathrm{mm}$ instrument channel and $30^{\circ}$ oblique forward viewing optics. An electronic convex array ultrasound transducer was mounted at the distal tip and was covered by a water inflatable balloon sheath. Scanning was performed at a frequency of $7.5 \mathrm{MHz}$ and with a penetration of $50 \mathrm{~mm}$. The angle of view was $90^{\circ}$ and the direction of view was $30^{\circ}$ forward oblique.

A dedicated 21/22-gauge needle (XNA-202C; Olympus, Ltd.) was developed to perform the TBNA. The needle was also equipped with a stylet that was withdrawn subsequent to passing the bronchial wall, avoiding contamination during the TBNA. The needle was mounted at the biopsy channel inlet of the endoscope prior to puncture and the needle exited the outer covering of the insertion tube at $20^{\circ}$. The needle was visualized through the optics and on the ultrasound image.

Biopsy procedure. The probe was introduced through the mouth and vocal cords into the main carina. The balloon was partially inflated (0.3-0.5 ml water) and the regional lymph node stations of the middle mediastinum and hilar regions (stations 2, 3, 4, 7, 10 and 11) were systematically imaged and measured (short-axis diameter) during the slow withdrawal and rotation of the transducer. Fine needle aspiration (FNA) was performed by passing the dedicated prototype 21/22-gauge needle through the airway wall and into the lymph nodes under real-time ultrasound guidance. Needle punctures were performed using the jabbing method. Integrated power Doppler ultrasound was used to visualize and avoid potentially intervening vessels immediately prior to needle puncture.
One to four mediastinal lymph nodes were sampled per patient, with a maximum of seven passes/node. EBUS-TBNA aspirates were expelled from the needle by either blowing air through a $20-\mathrm{ml}$ syringe or by reinsertion of the stylet. A portion of the aspirates was smeared on glass slides, air-dried and immediately stained with a Diff-Quick (DQ) staining kit (NC9943455; Fisher Scientific, Pittsburgh, PA, USA). Another portion of the aspirates was also smeared on glass slides and immediately placed into 95\% ethanol for Papanicolaou (Pap) staining. The remaining specimen, collected subsequent to rinsing with Cytorich Red (05NG-00003; BD Diagnostics, Burlington, NC, USA), was collected in a Cytorich-containing cup and processed for ThinPrep (BD PrepStain Slide Processor; BD Diagnostics) and cell block evaluation. The last two to five aspirates of EBUS-TBNA specimens/site were collected and placed directly into Cytorich Red for additional studies, including cell block preparations. These were not examined on-site. The sediment obtained from the Cytorich Red cup was processed in paraffin blocks, and the histological slides were stained with hematoxylin-eosin. Up to five passes/lymph node were collected, smeared and examined on-site. However, the procedure was terminated earlier if a tumor/granuloma or other specific diagnoses were reached.

Acid-Fast Bacilli (AFB) and Gomori-Grocott's methenamine silver stains were performed in the cell block slides of all samples with granuloma. In certain cases, culture of Mycobacterium tuberculosis was also conducted. Immunostaining was conducted at the discretion of the attending pathologists.

Final diagnosis. Lymph node aspirates obtained by EBUS-TBNA were considered to be representative if two lymphohistiocytic aggregates or five groups of lymphocytes ( $>20$ cells/group) were identified. Aspirates containing other cellular material that resulted in a specific diagnosis (carcinoma, thymoma, granulomas or lymphoma) were also considered representative (8).

The diagnosis of tumors from EBUS-TBNA was considered final. When a granuloma was identified, a diagnosis of mycobacteria infection was explored by AFB stain and/or bacterial culture. The final diagnosis of sarcoidosis was based on clinical and radiological suspicion, tissue confirmation of noncaseating granulomas and a follow-up period, subsequent to which similarly presenting diseases, such as lung cancer, lymphoma or tuberculosis, could be excluded.

Additional tissue examination of a particular patient was based wholly on clinical suspicion/treatment decisions. Whenever possible, the tissue diagnosis was compared with that derived from the EBUS-TBNA aspirates. Reactive lymph nodes diagnosed by biopsy were considered true negative results; tumors (including lymphoma) and sarcoidosis diagnosed by biopsy/clinical follow-up were considered true positive results.

Statistical methods. The primary end-point of the study was the percentage of biopsy specimens obtained that contained lymphocytes/lymphohistiocytic aggregates. The secondary end-point was the percentage of confirmed diagnoses made possible with EBUS-TBNA. Diagnostic sensitivity, specificity and accuracy were calculated using the standard definitions: 
The proportion of true positive results, true negative results and all correct results, respectively. Reactive lymph nodes diagnosed by biopsy were considered true negative results; tumors (including lymphoma) and sarcoidosis diagnosed by biopsy/clinical follow-up were considered true positive results. The unit of analysis was the patient.

\section{Results}

Patient demographics. EBUS-TBNA was performed in all 51 patients; the majority of the patients (29 patients, $57 \%$ ) were of of African-American descent. The average age of the patients was 57.1 years. On-site smear preparation and interpretation, as well as cell block preparation, were attempted in all patients.

Learning analysis. The retrospective analysis showed that, in the first 8 months, tumor/granuloma diagnoses were made in only $9.1 \%$ of cases [three nodes/masses, one lymphoma, one thymoma and one squamous cell carcinoma (SCC)] while specimens that were negative for malignant cells comprised $84.9 \%$ of cases. By contrast, tumor/granuloma diagnoses were achieved in $27 \%$ of cases in the second 8 months (21 nodes, 10 pulmonary adenocarcinoma, two SCCs, one non-classified poorly differentiated carcinoma and eight granulomas) $(\mathrm{P}=0.045$ versus the first 8 months) (Table I).

In the first 8 months, cell blocks could be made in only 14 out of 33 lymph nodes/masses $(42.4 \%)$ due to poor cellularity in the remaining cases. By contrast, cell blocks were available in 71 out of 79 cases in the second 8 months $(90.0 \%)$ $(\mathrm{P}<0.001$ versus the first 8 months $)$. Smear preparations (including DQ, Pap smear and thin preparations) were deemed as non-diagnostic samples in two out of 33 nodes/masses (6.1\%) in the first 8 months of the EBUS-TBNA procedure; in the second 8 months the node-diagnostic smear samples decreased to three out of 79 nodes $(3.8 \%)(\mathrm{P}=0.46$ versus the first 8 months) (Table I).

Correlation between EBUS-TBNA and biopsy. In the first 8 months, tissue diagnoses were available for 17 nodes/masses. Fourteen of the diagnoses were benign/reactive lymph nodes, and the corresponding FNA diagnoses were $100 \%$ matched with the tissue diagnoses. There were three tumor diagnoses by tissue examination: SCC, thymoma and B-cell lymphoma. The corresponding FNA diagnoses were SCC, atypical lymphoid tissue and suspicious for thymoma, respectively. In the second 8 months, tissue diagnoses were available for 23 nodes/masses, including three adenocarcinomas, two SCCs and seven granulomas, representing 33.0, 100 and $88.0 \%$ of the total EBUS-TBNA case numbers in their respective category, respectively. The eight benign/reactive diagnoses of the biopsy showed $100 \%$ concordance with the FNA diagnoses. There were 12 tumor/noncaseating granuloma diagnoses by tissue examination, three adenocarcinomas, two SCCs and eight noncaseating granulomas. The corresponding FNA diagnoses were $100 \%$ matched with the tissue diagnoses (Figs. 1 and 2). The sensitivity, specificity and accuracy in the first 8 months were $33.0,100$ and $88.0 \%$, respectively. The sensitivity, specificity and accuracy in the second 8 months were all $100 \%$ (Fig. 3).
Table I. Comparison of sample adequacy and diagnostic accuracy over time.

\begin{tabular}{lccr}
\hline & $\begin{array}{c}\text { First } \\
8 \text { months, } \\
\text { n/total n (\%) }\end{array}$ & $\begin{array}{c}\text { Second } \\
\text { 8 months, }\end{array}$ & \\
n/total n (\%) & P-value \\
\hline Cell block & $14 / 33(42)$ & $71 / 99(90)$ & $<0.001$ \\
Tumor/granuloma & $3 / 33(9)$ & $21 / 79(27)$ & 0.045 \\
Non-diagnostic smears & $2 / 33(6)$ & $3 / 79(4)$ & 0.460 \\
$\begin{array}{l}\text { FNA versus tissue correlation } \\
\text { for tumor/granuloma }\end{array}$ & $1 / 3(33)$ & $12 / 12(100)$ & 0.029 \\
\hline
\end{tabular}

FNA, fine needle aspiration.

Immunohistochemical and genetic prognostic marker analysis. Immunohistochemical stains were performed in cell blocks of 10 tumor samples: Seven adenocarcinomas and three SCCs. All of the adenocarcinomas were thyroid transcription factor-1 (TTF-1)-positive with negative or very focal p63 stains; all of the SCCs were TTF-1-negative and p63- and cytokeratin 5/6 (CK5/6)-positive (Figs. 1 and 2).

EGFR/KRAS mutation analysis was conducted in the cell blocks of five adenocarcinomas upon the request of the treating physicians. All of the cell blocks contained sufficient material for analysis; one case showed a mutation in exons 18-21 of EGRF, two cases revealed KRAS mutations at codons 12 and 13 and two cases exhibited no EGFR or KRAS mutation.

Complications. No sedative drugs were administered to any patients during the procedure. No major complications, such as pneumothorax, mediastinal emphysema or bleeding from ruptured major vessels in the mediastinum, were noted during the procedure or in the follow-up period.

\section{Discussion}

This study is not the first to report a learning curve following the initial application of EBUS-TBNA in a medical center (7). Groth et al (9) suggested that the learning curve for EBUS-FNA for thoracic surgeons is $\sim 10$ procedures. Sun et al (10) reported that, following the initial five procedures, the sensitivity of EBUS-TBNA for diagnosing lung cancer should be $\geq 90 \%$ for pulmonologists experienced in bronchoscopy. However, the majority of these reports are from tertiary medical centers with a selected patient population. At least one recent study has suggested that EBUS-TBNA results from these centers may not be entirely representative in a more general patient population in routine care (11). The present study was conducted in an urban community medical center, with the patient population including 57\% African-Americans and a significant number of patients who were human immunodeficiency virus (HIV)-positive. In this medical center, diagnoses of 13 out of the first 16 patients were benign (no evidence of malignant cells). Four of these 13 patients underwent biopsy/resection and $100 \%$ agreement between TBNA and the histological diagnoses was achieved. The first EBUS-TBNA diagnosis of metastatic pulmonary carcinoma was made in the 16th patient, which was confirmed later by an evaluation of a histological biopsy 


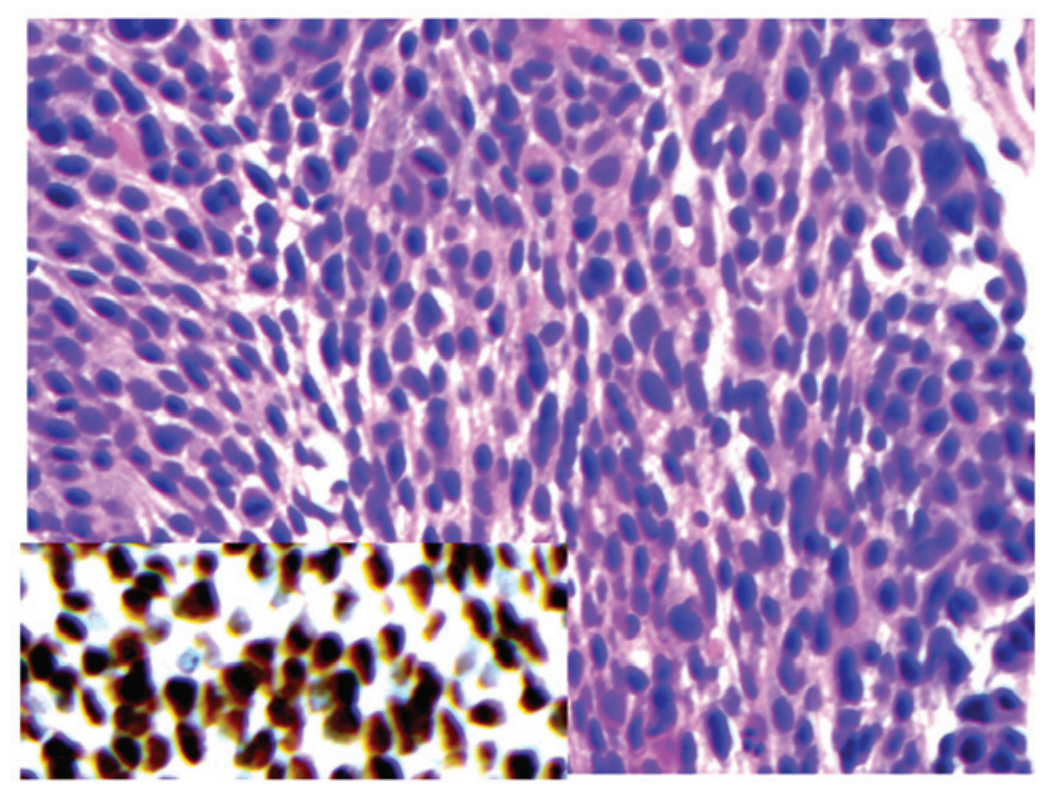

Figure 1. Adenocarcinoma of the lung (magnification, $x$ 400). Insert: thyroid transcription factor-1 immunostain.

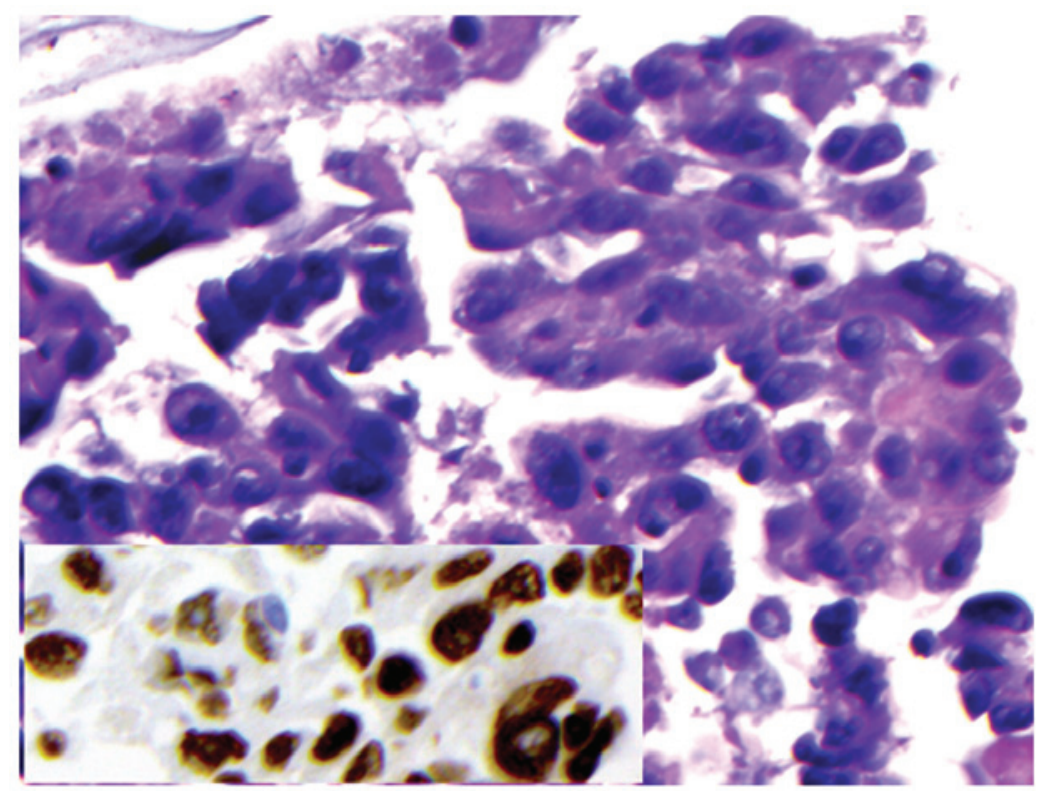

Figure 2. Squamous cell carcinoma (magnification, x 400). Insert: p63 immunostain.

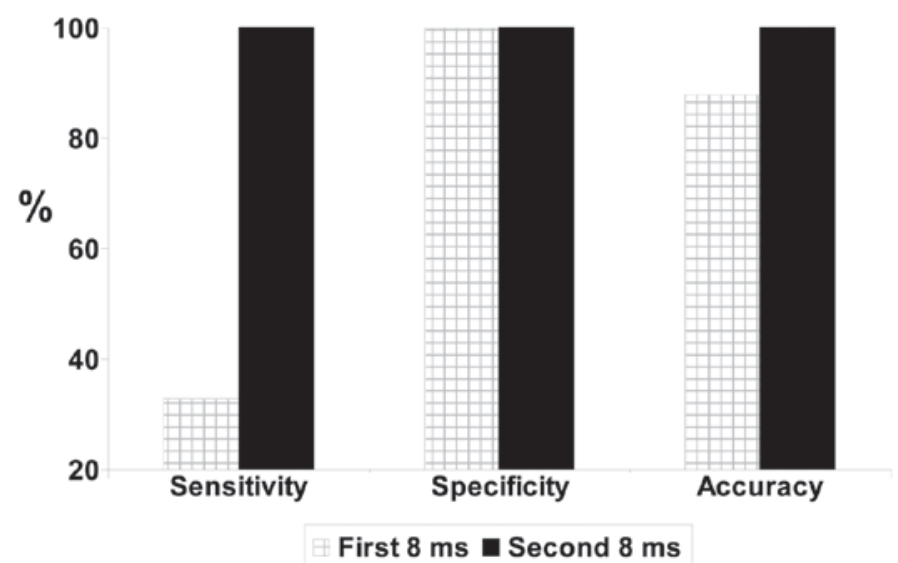

Figure 3. Learning curve for endobronchial ultrasound-guided transbronchial needle aspiration. ms, months. 
sample. The two misclassified tumors (one thymoma and one B-cell lymphoma) occurred in the third and eighth patients, respectively. It is important to note that both false positive and false negative results have been reported for EBUS-TBNA, even in centers experienced in this procedure (12). Limited cellularity is clearly a contributing factor to the difficulty in classification, as the aspirated material from one of three tumors was not sufficient to make a cell block (the thymoma case); the material from another patient (the lymphoma case) was not sufficient to run flow cytometry. In the first 8 months of this study, cell blocks were made in only $42 \%$ of cases due to poor cellularity. Rapid on-site evaluation (ROSE) and effective communication with the bronchoscopists are therefore critical to guarantee sufficient cellularity and achieve a correct diagnosis. As suggested by Monaco et al (13), the presence of cytologists also allows the appropriate triage of material for ancillary studies, including tissue culture, flow cytometry, immunostaining and molecular studies. At the initial stage of the EBUS-TBNA procedure, a lack of standardization of the ROSE procedure and the different expections from bronchoscopists, pathologists and radiologists may also contribute to the suboptimal early results.

The tumor/granuloma diagnoses were significantly increased to $27 \%$ in the second 8 months of the study. Sufficient diagnostic material in the majority of cases (cell blocks in $90 \%$ of cases) and effective communication with bronchoscopists (ROSE) were necessary for this improvement. Other factors, including accurate positioning of the probe into the lesion following further practice (14), may also have influenced the improvement. The pattern of the learning curve for this EBUS-TBNA is similar to that of previous reports $(5,8,9,15)$. However, significant differences between our results and previous reports were also noted. Firstly, the tumor diagnosis rate in the cohort in the second 8 months was $16 \%$, less than that of previous reports $(\sim 45 \%)(2,5)$. Secondly, the rate of sarcoidosis was $10 \%$, which was higher than that reported in previous studies $(\sim 5 \%)$. In addition, the malignancy rate in the second 8 months was $71 \%$, which was higher than that reported in previous studies $(\sim 35 \%)$. The majority of these differences may be attributed to different patient populations due to the following reasons: i) There was no significant increase in the tumor/granuloma diagnostic rate in the center, even during the most recent months (after the second 8 months); ii) a recent study using a population under routine care also achieved a similar tumor/sarcoidosis diagnostic rate of $27 \%$ (11); iii) a significant number of patients in our medical center immunosuppressive (patients who had undergone heart, lung or renal transplantations and patients with HIV) (16). Rates of infection and associated mediastinal reactive lymphadenopathy were therefore expected to be higher than those in previously reported patient populations.

With the advances in personalized chemotherapy, the subclassification of non-small cell lung carcinomas has become necessary. The EGFR inhibitors erlotinib and gefitinib are most efficacious in tumors with EGFR mutation, which are predominantly adenocarcinomas (17). Similarly, the folate inhibitor pemetrexed is currently the most effective drug treatment for adenocarcinoma, but not SCC (18). An inhibitor of anaplastic lymphoma kinase (ALK), crizotinib, targets tumors with EML4-ALK fusion, which occurs predominantly in adenocarcinoma (19). One caveat of the EBUS-TBNA specimen is the limited diagnostic material, which requires the strategic use of antibodies rather than screening with a panel of antibodies. It is noteworthy that in the present analyzed cohort, only two or three antibodies were required to differentiate the non-small cell carcinomas of the pulmonary origin. In this study, immunohistochemical stains were performed in the cell blocks of seven metastatic carcinomas of the pulmonary origin (four adenocarcinomas and three SCCs). All the adenocarcinomas were TTF-1-positive with negative or very focal p63 stains and the SCCs were TTF-1-negative and extensively positive for p63 and CK5/6. These results closely correlated with the conclusions by Mukhopadhyay (20) from studies of small histology biopsy samples. The analysis of genetic mutations to EGFR, Ras and ALK is requied for the effective treatment of pulmonary adenocarcinoma, either for the selection of chemotherapy or due to the selection criteria for clinical trials. In all the cases of pulmonary adenocarcinoma, EGFR and KRAS mutation analyses were successfully performed.

The findings from this study indicated a significant difference between the cytological results from the early and late case groups, suggesting that a steep learning curve should be expected when EBUS-TBNA is first adopted in a medical center. Every attempt should be made to prepare cell blocks, which are critical for the subclassification of tumors and possibly for the assessment of therapeutic and prognostic markers. TTF-1, p63 and possibly CK5/6 are usually sufficient to differentiate SCC from adenocarcinoma of the lung.

\section{References}

1. Varela-Lema L, Fernández-Villar A and Ruano-Ravina A: Effectiveness and safety of endobronchial ultrasound-transbronchial needle aspiration: a systematic review. Eur Respir J 33: 1156-1164, 2009.

2. Navani N, Lawrence DR, Kolvekar S, Hayward M, McAsey D, Kocjan G, Falzon M, Capitanio A, Shaw P, Morris S, Omar RZ and Janes SM; REMEDY Trial Investigators: Endobronchial ultrasound-guided transbronchial needle aspiration prevents mediastinoscopies in the diagnosis of isolated mediastinal lymphadenopathy: a prospective trial. Am J Respir Crit Care Med 186: 255-260, 2012.

3. Oki M, Saka H, Kitagawa C, Kogure Y, Murata N, Ichihara S, and MoritaniS: Prospective study of endobronchial ultrasound-guided transbronchial needle aspiration of lymph nodes versus transbronchial lung biopsy of lung tissue for diagnosis of sarcoidosis. J Thorac Cardiovasc Surg 143: 1324-1329, 2012.

4. Gurioli C. Ravaglia C, Romagnoli M, Casoni G, Tomassetti S, Nanni $\mathrm{O}$ and Poletti V: EBUS-TBNA in mediastinal/hilar lymphadenopathies and/or masses: an Italian case series. Clin Respir J 6: 3-8, 2012.

5. Delattre C, Fournier C, Bouchindhomme B, Renaud F, Escande F, Ramon P and Copin MC: Endoscopic ultrasound guided transbronchial fine needle aspiration: a French Department of Pathology's 4-year experience. J Clin Pathol 64: 1117-1122, 2011.

6. Herth FJ, Eberhardt R, Vilmann P, Krasnik M and Ernst A: Real-time endobronchial ultrasound guided transbronchial needle aspiration for sampling mediastinal lymph nodes. Thorax 61: 795-798, 2006.

7. Wallace MB, Pascual JM, Raimondo M, Woodward TA, McComb BL, Crook JE, Johnson MM, Al-Haddad MA, Gross SA, Pungpapong S, Hardee JN and Odell JA: Minimally invasive endoscopic staging of suspected lung cancer. JAMA 299: 540-546, 2008

8. Nayak A, Sugrue C, Koenig S, Wasserman PG, Hoda S and Morgenstern NJ: Endobronchial ultrasound-guided transbronchial needle aspirate (EBUS-TBNA): a proposal for on-site adequacy criteria. Diagn Cytopathol 40: 128-137, 2012. 
9. Groth SS, Whitson BA, D'Cunha J, Maddaus MA, Alsharif M and Andrade RS: Endobronchial ultrasound-guided fine-needle aspiration of mediastinal lymph nodes: a single institution's early learning curve. Ann Thorac Surg 86: 1104-1110, 2008.

10. Sun JY, Zhao H, Zhang J, Wang XD and Han BH: First 30 endobronchial ultrasound-guided transbronchial needle aspirations: a single institution's early experience. Chin Med J (Engl) 124: $1818-1823,2011$.

11. Lange TJ, Kunzendorf F, Pfeifer M, Arzt M and Schulz C: Endobronchial ultrasound-guided transbronchial needle aspiration in routine care - plenty of benign results and follow-up tests. Int J Clin Pract 66: 438-445, 2012.

12. Sanz-Santos J, Andreo F, Serra P, Llatjós M, Castellà E, Astudillo J, Monsó E and Ruiz-Manzano J: False positive endobronchial ultrasound-guided real-time transbronchial needle aspiration secondary to bronchial carcinoma in situ at the point of puncture: a case report. J Cardiothorac Surg 7: 74, 2012.

13. Monaco SE, Pantanowitz L and Khalbuss WE: Comparing endobronchial ultrasound-guided fine needle aspiration specimens with and without rapid on-site evaluation. Cytojournal 9: 2, 2012

14. Huang CT, Tsai YJ, Liao WY, Wu PC, Ho CC, Yu CJ and Yang PC: Endobronchial ultrasound-guided transbronchial biopsy of peripheral pulmonary lesions: how many specimens are necessary? Respiration 84: 128-134, 2012.

15. Lee BE, Kletsman E, Rutledge JR and Korst RJ: Utility of endobronchial ultrasound-guided mediastinal lymph node biopsy in patients with non-small cell lung cancer. J Thorac Cardiovasc Surg 143: 585-590, 2012.
16. Vaughn AC: The impact of substance abuse on the care of African Americans and Latinos with HIV/AIDS. J Natl Med Assoc 96 (2 Suppl): 21S-23S, 2004.

17. Shigematsu H, Lin L, Takahashi T, Nomura M, Suzuki M, Wistuba II, Fong KM, Lee H, Toyooka S, Shimizu N, Fujisawa T, Feng Z, Roth JA, Herz J, Minna JD and Gazdar AF: Clinical and biological features associated with epidermal growth factor receptor gene mutations in lung cancers. J Natl Cancer Inst 97: 339-346, 2005.

18. Scagliotti GV, Parikh P, von Pawel J, Biesma B, Vansteenkiste J, Manegold C, Serwatowski P, Gatzemeier U, Digumarti R, Zukin M, Lee JS, Mellemgaard A, Park K, Patil S, Rolski J, Goksel T, de Marinis F, Simms L, Sugarman KP and Gandara D: Phase III study comparing cisplatin plus gemcitabine with cisplatin plus pemetrexed in chemotherapy-naive patients with advanced-stage non-small-cell lung cancer. J Clin Oncol 26: 3543-3551, 2008.

19. Kwak EL, Bang YJ, Camidge DR, Shaw AT, Solomon B, Maki RG, Ou SH, Dezube BJ, Jänne PA, Costa DB, Varella-Garcia M, Kim WH, Lynch TJ, Fidias P, Stubbs H, Engelman JA, Sequist LV, Tan W, Gandhi L, Mino-Kenudson M, Wei GC, Shreeve SM, Ratain MJ, Settleman J, Christensen JG, Haber DA, Wilner K, Salgia R, Shapiro GI, Clark JW and Iafrate AJ: Anaplastic lymphoma kinase inhibition in non-small-cell lung cancer. $\mathrm{N}$ Engl J Med 363: 1693-1703, 2010.

20. Mukhopadhyay S: Utility of small biopsies for diagnosis of lung nodules: doing more with less. Mod Pathol 25 (Suppl 1): S43-S57, 2012. 